\title{
MINIMAL MODELS OF NILMANIFOLDS
}

\author{
KEIZO HASEGAWA \\ (Communicated by David G. Ebin)
}

\begin{abstract}
In this paper we first determine minimal models of nilmanifolds associated with given rational nilpotent Lie algebras. Then we study some properties of nilmanifolds through their associated Lie algebras and minimal models. In particular, we will see that a minimal model of a nilmanifold is formal if and only if it is a torus, and thus a non-toral nilmanifold has no complex structure which is birationally isomorphic to a Kähler manifold.
\end{abstract}

\section{INTRODUCTION}

A nilmanifold is a compact homogeneous space of nilpotent Lie group. The nilmanifolds are known to give counterexamples relating to Kähler structure: non-Kähler almost Kähler manifolds, non-Kähler symplectic manifolds, compact complex manifolds of which the Frölicher spectral sequence does not degenerate at $E^{1}$, and so forth.

There is a series of papers in this area starting with Thurston's paper [12] on non-Kähler symplectic manifolds (cf. $[1,4,5,7,10]$ ).

In this paper, instead of some specific nilmanifolds, we discuss general nilmanifolds in terms of their associated rational nilpotent Lie algebras. This way clarifies as well as generalizes the arguments in the related problems; for instance, Kodaira-Thurston's first example of non-Kähler symplectic manifold can be characterized as a 4-dimensional nilmanifold with its associated nilpotent Lie algebra $\mathfrak{g}$, where $\mathfrak{g}$ has a basis $\left\{X_{1}, X_{2}, X_{3}, X_{4}\right\}$ for which the only nonzero bracket multiplication is $\left[X_{1}, X_{2}\right]=-X_{3}$.

In $\S 1$ we determine explicitly minimal models of nilmanifolds associated with given rational nilpotent Lie algebras. In $\S 2$ it will be shown that a minimal model of a nilmanifold is formal if and only if it is a torus. Applying a result of Deligne, Griffiths, Morgan, and Sullivan [6], we see that a non-toral nilmanifold has no birational Kähler structure (see $\S 2$ for definition). In $\S 3$ we briefly discuss symplectic structures of nilmanifolds through their associated Lie algebras and

Received by the editors September 10, 1987 and, in revised form, June 10, 1988.

1980 Mathematics Subject Classification (1985 Revision). Primary 14M17, 32M10, 53C30; Secondary $14 \mathrm{M} 17,53 \mathrm{C} 15,57 \mathrm{~T} 20$.

Key words and phrases. Rational nilpotent Lie algebra, formality of minimal model, symplectic structure, Kähler structure. 
minimal models. In particular, we see that a non-toral nilmanifold has no invariant symplectic structure.

\section{MiNimal MODElS OF NILMANIFOLDS}

Following Sullivan (cf. [6]),we briefly review $K$-minimal models for commutative differential graded algebras over $K$, where $K$ is $\mathbf{Q}$ or $\mathbf{R}$.

Let $A$ be a commutative differential graded algebra over $K$. This means that $A$ is a graded algebra over $K$ :

$$
A=\bigoplus_{i \geq 0} A^{i}
$$

with the commutativity:

$$
a b=(-1)^{p q} b a \quad\left(a \in A^{p}, b \in A^{q}\right) ;
$$

and an antiderivation $d$ of degree 1:

$$
d^{2}=0, \quad d(a b)=(d a) b+(-1)^{p} a(d b) \quad\left(a \in A^{p}, b \in A^{q}\right) .
$$

Let $B$ be another differential graded algebra over $K . B$ is a Hirsch extension of degree $n$ of $A$, if $B$ is of the following form:

$$
\begin{aligned}
& B=A \otimes \Lambda_{n}\left\langle x_{1}, x_{2}, \ldots, x_{k}\right\rangle, \operatorname{deg} x_{i}=n, d x_{i} \in A \text { for } i= \\
& 1,2, \ldots, k
\end{aligned}
$$

where $\Lambda_{n}\left\langle x_{1}, \ldots, x_{k}\right\rangle$ is the exterior algebra generated by $x_{1}, x_{2}, \ldots, x_{k}$.

A commutative differential graded algebra $A$ over $K$ is minimal if $A$ satisfies the following conditions:

(1) $A=\bigcup_{i \geq 0} A_{i}$, where $A_{0}=K$, and $A_{i+1}$ is a Hirsch extension of $A_{i}$ for $i \geq 0$.

(2) $d x \in A_{+} \cdot A_{+}$for all $x \in A$, where $A_{+}=\bigoplus_{i \geq 1} A^{i}$.

A commutative differential graded algebra $\mathscr{A}$ over $K$ is a $K$-minimal model for $A$, if $\mathscr{A}$ is minimal and there exists a morphism $\phi$ of differential graded algebras from $\mathscr{A}$ to $A$ such that $\phi$ induces an isomorphism on cohomology.

A $K$-minimal model $\mathscr{M}$ of a differentiable manifold $M$ is a $K$-minimal model for the $K$-De Rham complex $\varepsilon^{*}(M)$ of $M$. The Q-De Rham complex is defined by Q-polynomial forms instead of usual smooth forms (cf. [2, 6]).

Now, let $M$ be a nilmanifold. According to Malcev [9], $M$ can be written as $G / \Gamma$, where $G$ is a simply connected nilpotent Lie group and $\Gamma$ is a uniform discrete subgroup of $G$. Let $\mathfrak{g}$ be the Lie algebra of $G$ (the vector space of all $G$-right invariant vector fields on $G$ ). Malcev proved the following fundamental theorem on nilmanifolds: $\mathfrak{g}$ has a basis for which the structure constants are all rational; and conversely for every nilpotent Lie algebra $\mathfrak{g}$ with rational constants of structure, there exists a nilmanifold $M$ with $\mathfrak{g}$ as its associated Lie algebra. 
Let us consider the following commutative differential graded algebras over $\mathbf{R}$ :

$$
\begin{aligned}
C(M) & =\bigoplus_{q \geq 0} C^{q}(M), C^{q}(M)=\{w: w \text { is a } \Gamma \text {-right invariant } q \text {-form on } G\}, \\
C(G) & =\bigoplus_{q \geq 0} C^{q}(G), C^{q}(G)=\{w: w \text { is a } G \text {-right invariant } q \text {-form on } G\}, \\
C(\mathfrak{g}) & =\bigoplus_{q \geq 0} C^{q}(\mathfrak{g}), C^{q}(\mathfrak{g})=\{f: f \text { is a } q \text {-linear alternating function on } \mathfrak{g}\},
\end{aligned}
$$

where $C(M)$ can be identified with the R-De Rham complex $\varepsilon^{*}(M)$.

According to the theorem of Koszul (cf. [3]), there exists a canonical isomorphism from $H^{*}(\mathfrak{g})$ to $H^{*}(G)$. Let $k=\operatorname{dim} H^{1}(\mathfrak{g})=\operatorname{dim}(\mathfrak{g} /[\mathfrak{g}, \mathfrak{g}])$. Since $g$ is a nilpotent Lie algebra over $\mathbf{Q}$, there exists a basis $\left\{X_{1}, X_{2}, \ldots, X_{m}\right\}$ for which $[\mathfrak{g}, \mathfrak{g}]=\left\langle X_{k+1}, \ldots, X_{m}\right\rangle$ and $\left[X_{i}, X_{j}\right]=-\sum_{j<p} a_{p i j} X_{p}(i<j)$, where $m=\operatorname{dim} g, m \geq k \geq 2$, and $a_{p i j} \in \mathbf{Q}$.

Let $w_{1}, w_{2}, \ldots, w_{m}$ be the Maurer-Cartan forms on $G$ ( $G$-right invariant 1-forms) which correspond to $X_{1}, X_{2}, \ldots, X_{m}$ respectively. Then the forms $w_{1}, w_{2}, \ldots, w_{m}$ satisfy the following formulas:

$$
\begin{gathered}
d w_{p}=\sum_{i<j<p} a_{p i j} w_{i} \wedge w_{j} ; \quad \text { where } d w_{p}=0 \text { for } p \leq k, \\
\text { and } d w_{p} \neq 0 \text { for } p>k .
\end{gathered}
$$

Since $C^{0}(G)$ consists of constant functions on $G$, none of $w_{1}, w_{2}, \ldots, w_{m}$ are exact in $C(G)$. Hence an R-minimal model $\widehat{\mathscr{M}}$ for $C(G)$ is the following:

$$
\widehat{\mathscr{M}}=\widetilde{\mathscr{M}} \otimes \mathbf{R},
$$

where $\widetilde{\mathscr{M}}=\Lambda_{1}\left\langle x_{1}, x_{2}, \ldots, x_{m}\right\rangle$ is the commutative differential graded algebra over $\mathbf{Q}$, generated by $x_{1}, x_{2}, \ldots, x_{m}, \operatorname{deg} x_{i}=1(i=1, \ldots, m)$, and the antiderivation $d$ on $\widetilde{\mathscr{M}}$ is uniquely determined by (1.1). Remark that the condition, $d^{2}=0$, follows from the Jacobi identity in $\mathfrak{g}$ (cf. [3]).

The Q-minimal differential graded algebra $\widetilde{\mathscr{M}}$ can be written inductively as follows:

$$
\begin{aligned}
& \widetilde{\mathscr{M}}=\widetilde{\mathscr{M}}_{m}, \widetilde{\mathscr{M}}_{s+1}=\widetilde{\mathscr{M}}_{s} \otimes \Lambda_{1}\left\langle x_{s+1}\right\rangle, d x_{s+1} \in \widetilde{\mathscr{M}}_{s}^{1} \cdot \widetilde{\mathscr{M}}_{s}^{1}, d x_{s+1} \neq 0 \\
& \text { for } s(m-1 \geq s \geq k), \text { and } \widetilde{\mathscr{M}}_{k}=\Lambda_{1}\left\langle x_{1}, x_{2}, \ldots, x_{k}\right\rangle, d x_{1}= \\
& d x_{2}=\cdots=d x_{k}=0, \quad k \geq 2 .
\end{aligned}
$$

According to the theorem of Nomizu [11], the canonical morphism of differential graded algebras from $C(G)$ to $C(M)$ induces an isomorphism from $H^{*}(G)$ to $H^{*}(M)$. Therefore, $\widehat{\mathscr{M}}$ is an R-minimal model of $M$. 
Remark. $\widetilde{\mathscr{M}}$ is actually a Q-minimal model of $M$ (cf. [6]). Conversely, it can be seen that for a Q-minimal differential graded algebra $\widetilde{\mathscr{M}}$ of the form above there exists a nilmanifold $M$ with $\widetilde{\mathscr{M}}$ as its Q-minimal model.

\section{FORMALITY OF MINIMAL MODEL}

A minimal differential graded algebra $A$ over $K$ is formal if there exists a morphism $\psi$ of differential graded algebras from $A$ to $H^{*}(A ; K)$ such that $\psi$ induces the identity on cohomology, with the antiderivation $d=0$ on $H^{*}(A ; K)$.

Let $\widetilde{\mathscr{M}}$ be a Q-minimal model of a nilmanifold $M$ of dimension $m$.

Lemma 1. $d \widetilde{\mathscr{M}}^{m-1}=0$, and thus $\operatorname{dim} H^{m}(\widetilde{\mathscr{M}} ; \mathbf{Q})=1$.

Proof. Since $\left\{x_{1} x_{2} \cdots \hat{x}_{q} \cdots x_{m}\right\} \quad(q=1,2, \ldots, m)$ forms a basis of $\widetilde{\mathscr{M}}^{m-1}$, it is sufficient to show that $d\left(x_{1} x_{2} \cdots \hat{x}_{q} \cdots x_{m}\right)=0$ for $q=1,2, \ldots, m$. But this is obvious because $d x_{s+1} \in \widetilde{\mathscr{M}}_{s}^{1} \cdot \mathscr{\mathscr { M }}_{s}^{1}$ for $s=k, k \ldots, m-1$ and $d x_{s}=0$ for $s=1, \ldots, k(k \geq 2)$.

Theorem 1. $\widetilde{\mathscr{M}}$ is formal if and only if $M$ is a torus.

Proof. A nilmanifold $M$ is a torus if and only if $G$ is Abelian; this is because a simply connected Abelian Lie group is isomorphic to $\mathbf{R}^{m}$, and any discrete subgroup of $\mathbf{R}^{m}$ is a lattice. $G$ is Abelian if and only if its Lie algebra $g$ is Abelian, which is the case $k=m$, where $k=\operatorname{dim} H^{1}(\widetilde{\mathscr{M}} ; \mathbf{Q})=\operatorname{dim} H^{1}(M ; \mathbf{Q})$. It is therefore sufficient to show that $\widetilde{\mathscr{M}}$ is formal if and only if $k=m$.

Suppose that $k<m$ and $\widetilde{\mathscr{M}}$ is formal. Then there exists a morphism $\psi$ of differential graded algebras from $\widetilde{\mathscr{M}}$ to $H^{*}(\widetilde{\mathscr{M}} ; \mathbf{Q})$ which induces the identity on cohomology. Let us consider the restriction $\psi^{1}$ of $\psi$ to $\widetilde{\mathscr{M}}^{1}$. Since $\psi$ induces the identity on cohomology, $\psi$ maps every closed form of $\tilde{\mathscr{M}}$ to its cohomology class. In particular, $\psi^{1}\left(x_{q}\right)=\left[x_{q}\right]$ in $H^{1}(\widetilde{\mathscr{M}} ; \mathbf{Q})$ for $q=1,2 \ldots, k$. Since $\left\{\left[x_{q}\right]\right\}(q=1,2, \ldots, k)$ forms a basis of $H^{1}(\widetilde{\mathscr{M}} ; \mathbf{Q}), \widetilde{\mathscr{M}}^{1}$ is generated by $x_{1}, x_{2}, \ldots, x_{k}$ and a basis $\left\{y_{k+1}, \ldots, y_{m}\right\}$ of $\operatorname{Ker} \psi^{1}$. Now since $x_{1} x_{2} \cdots x_{m}=a x_{1} \cdots x_{k} y_{k+1} \cdots y_{m}$ for some nonzero scalar $a, \psi\left(x_{1} x_{2} \cdots x_{m}\right)=$ 0 . On the other hand, since $x_{1} x_{2} \cdots x_{m}$ is closed $\psi\left(x_{1} x_{2} \cdots x_{m}\right)=\left[x_{1} x_{2} \cdots x_{m}\right]$. But this contradicts Lemma 1 . Hence, $\widetilde{\mathscr{M}}$ is not formal unless $k=m$.

A compact complex manifold is birational Kähler if it is birationally isomorphic to a Kähler manifold. The birational Kähler manifold can be blown up to a Kähler manifold. According to the theorem of Deligne, Griffiths, Morgan, and Sullivan (cf. [6]), a $K$-minimal model of such a compact complex manifold is formal where $K$ is $\mathbf{Q}$ or $\mathbf{R}$. Therefore we obtain the following result.

Corollary. An even-dimensional nilmanifold has no birational Kähler structure unless it is a torus. 
Example 1. Let us consider a nilmanifold of dimension $(2 m+2)$ with its associated Lie algebra $\mathfrak{g}$, where $m \geq 1$ and $\mathfrak{g}$ has a basis

$$
\left\{X_{1}, \ldots, X_{m}, Y_{1}, \ldots, Y_{m}, Z, W\right\}
$$

for which the bracket multiplication of $\mathfrak{g}$ is defined as follows:

$$
\left[X_{i}, Y_{i}\right]=-Z(i=1, \ldots, m), \quad \text { and all other brackets are } 0 .
$$

An almost complex structure $J$ on $M$ is defined by

$$
\begin{aligned}
& J X_{i}=Y_{i}, \quad J Y_{i}=-X_{i}, \\
& J Z=W, \quad \text { and } J W=-Z, \quad \text { where } i=1, \ldots, m .
\end{aligned}
$$

The Nijenhuis tensor $N$ of this almost complex structure $J$ can be easily seen to vanish, where $N(X, Y)=[X, Y]+J[J X, Y]+J[X, J Y]-[J X, J Y]$. Thus $J$ defines a complex structure on $M$.

The first Betti number of $M$ is $2 m-1$. Remark that a complex surface with trivial canonical bundle in class $V I$ of Kodaira's classification of complex surfaces is a nilmanifold of the case $m=1$ in this example (cf. [8]).

Example 2. Let $M$ be a nilmanifold of dimension $(2 m+2)$ with its associated Lie algebra $\mathfrak{g}$, where $m \geq 2$ and $\mathfrak{g}$ has a basis $\left\{X_{1}, \ldots, X_{m}, Y_{1}, \ldots, Y_{m}\right.$, $Z, W\}$ for which the bracket multiplication of $\mathfrak{g}$ is defined as follows:

$$
\begin{aligned}
& {\left[X_{i}, Y_{i}\right]=-Z(i=1, \ldots, s),} \\
& {\left[X_{i}, Y_{i}\right]=-W(i=s+1, \ldots, m), \quad 1 \leq s<m,} \\
& \text { and all other brackets are } 0 .
\end{aligned}
$$

The almost complex structure $J$ on $M$ defined by (2.2) is integrable and thus defines a complex structure on $M$. The first Betti number of $M$ is $2 m$; furthermore, the odd-dimensional Betti numbers are all even.

Remark. A complex nilmanifold is, by definition, a nilmanifold of the form $G / \Gamma$, where $G$ is a simply connected complex nilpotent Lie group and $\Gamma$ is a uniform discrete subgroup of $G$. The examples above are not complex nilmanifolds: Suppose that there exists a complex structure $J$ on $\mathfrak{g}$. Then $\left[X_{1}, J Y_{1}\right]=J\left[X_{1}, Y_{1}\right]=-J Z$. Since $\left[X_{1}, \mathfrak{g}\right] \subset\langle Z\rangle$, it follows that $J Z=a Z$ for some real scalar $a$. But since $J^{2}=-1$, this is not possible.

\section{SYMPLECTIC STRUCTURES OF NILMANIFOLDS}

Let $M=G / \Gamma$ be a nilmanifold of dimension $m=2 n$ with its R-minimal model $\widehat{\mathscr{M}}$, where $G$ is a simply connected nilpotent Lie group and $\Gamma$ is a uniform discrete subgroup of $G$. The associated morphism $\phi$ of differential graded algebras from $\widehat{\mathscr{M}}$ to $\varepsilon^{*}(M)$ is uniquely determined by $\phi\left(x_{i}\right)=w_{i}(i=$ $1,2, \ldots, m)$, where $w_{1}, w_{2}, \ldots, w_{m}$ are the 1 -forms on $M$ defined by the Maurer-Cartan forms on $G$. Since $\Gamma$ is a discrete subgroup of $G, w_{1} \wedge w_{2} \wedge$ $\cdots \wedge w_{m}$ defines a volume element of $M$. In particular $M$ is orientable.

We say that $\widehat{\mathscr{M}}$ has a symplectic structure if it has a symplectic form $\omega$, closed 2-form such that $\omega^{n} \neq 0$. 
Lemma 2. $M$ has a symplectic structure if and only if $\widehat{\mathscr{M}}$ has a symplectic structure.

Proof. Suppose that $M$ has a symplectic structure. Then there exists a closed 2-form $\Omega$ on $M$ such that $\Omega^{n}$ is a volume element of $M$. Let $\omega$ be a closed

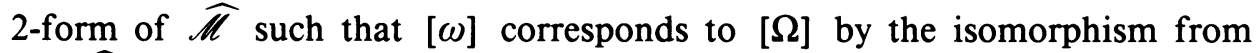
$H^{*}(\widehat{\mathscr{M}})$ to $H^{*}(M)$. Then clearly $[\omega]^{n} \neq 0$ and thus $\omega^{n} \neq 0$. Conversely, if has a symplectic form $\omega$, then $\Omega=\phi(\omega)$ is a symplectic form on $M$. This is because $\omega^{n}=a x_{1} x_{2} \cdots x_{m}(a \neq 0)$ and thus $\Omega^{n}=\phi\left(\omega^{n}\right)=a w_{1} \wedge w_{2} \wedge \cdots \wedge w_{m}$ (a volume element of $M$ ).

This lemma gives us a simple way to see if a given nilmanifold has a symplectic structure. For instance, let $M$ be a nilmanifold with its associated nilpotent Lie algebra $\mathfrak{g}$, where $\mathfrak{g}$ has a basis $\left\{X_{1}, X_{2}, X_{3}, X_{4}\right\}$ for which the only nonzero bracket multiplication is $\left[X_{1}, X_{2}\right]=-X_{3}$ (Kodaira-Thurston's example). Then by the result of $\S 1$, using the Maurer-Cartan's formula, an R-minimal model is the following:

$$
\widehat{\mathscr{M}}=\Lambda_{1}\left\langle x_{1}, x_{2}, x_{3}, x_{4}\right\rangle, \quad d x_{1}=d x_{2}=d x_{4}=0, \quad d x_{3}=x_{1} x_{2} .
$$

Clearly $\omega=x_{1} x_{3}+x_{2} x_{4}$ is a symplectic form of $\widehat{\mathscr{M}}$. Similarly we can construct many examples of symplectic nilmanifolds. However, concerning invariant symplectic structures of nilmanifolds we have the following result.

Theorem 2. An even-dimensional nilmanifold $M$ has no invariant symplectic structure unless $M$ is a torus.

Proof. By a $G$-invariant form on $M$ is meant a $G$-left invariant form on $M$, which can be identified with a $G$-left and $\Gamma$-right invariant form on $G$. According to a result of Nomizu [11], a $G$-left and $\Gamma$-right invariant form on $G$ is a $G$-left and $G$-right invariant form on $G$. Suppose that $M$ has an invariant symplectic form $\Omega$. Considering $\Omega$ as an element of $C(G)$, let $f$ be the element of $C(\mathfrak{g})$ corresponding to $\Omega$ by the canonical isomorphism from $C(G)$ to $C(\mathfrak{g})$, where $\mathfrak{g}$ is the Lie algebra of $G$. Since $f$ is closed, for any $X_{1}, X_{2}, X_{3}$ of $\mathfrak{g}, f\left(\left[X_{1}, X_{2}\right], X_{3}\right)+f\left(\left[X_{2}, X_{3}\right], X_{1}\right)+f\left(\left[X_{3}, X_{1}\right], X_{2}\right)=0$; but since $f$ is invariant, $f\left(\left[X_{2}, X_{3}\right], X_{1}\right)+f\left(\left[X_{3}, X_{1}\right], X_{2}\right)=0$. Hence, for any $X_{1}, X_{2}, X_{3}$ of $\mathfrak{g}, f\left(\left[X_{1}, X_{2}\right], X_{3}\right)=0$. The nondegeneracy of $f$ yields that $[\mathfrak{g}, \mathfrak{g}]=0$, that is, $\mathfrak{g}$ is Abelian. By the same argument as in Theorem 1 , this is the case that $M$ is a torus.

Note. I learned from Professor A. Gray that Cordero, Fernández and Gray showed, in their paper in preparation, the existence of a nonzero Massey product for a non-toral nilmanifold. I also learned that there is quite recently a preprint by Benson and Gordon (to appear in Topology) in which they showed that a non-toral nilmanifold has no Kähler structure.

\section{ACKNOWLEDGMENT}

This paper is partially based on the author's Ph.D. thesis (Berkeley, 1987). The author would like to express his sincere gratitude to Professors S. Kobayashi 
and $\mathrm{A}$. Weinstein for their kind advice and stimulating discussions to complete this paper.

\section{REFERENCES}

1. E. Abbena, An example of an almost Kähler manifold which is not Kählerian, Boll. Un. Mat. Ital. A(6) 3 (1984), 383-392.

2. H. Cartan, Théories cohomologiques, Invent. Math. 35 (1976), 261-271.

3. C. Chevalley and S. Eilenberg, Cohomology theory of Lie groups and Lie algebras, Trans. Amer. Math. Soc. 63 (1948), 85-124.

4. L. A. Cordero, M. Fernández and A. Gray, Symplectic manifolds with no Kähler structure, Topology 25 (1986), 375-380.

5. L. A. Cordero, M. Fernández and M. De Leon, Examples of compact non-Kähler almost Kähler manifolds, Proc. Amer. Math. Soc. 95 (1985), 280-286.

6. P. Deligne, P. Griffiths, J. Morgan and D. Sullivan, Real homotopy theory of Kähler manifolds, Invent. Math. 29 (1975), 245-274.

7. M. Fernández, M. J. Gotay and A. Gray, Compact parallelizable four-dimensional symplectic and complex manifolds (Preprint, 1986).

8. K. Kodaira, On the structure of compact complex analytic surfaces I, Amer. J. Math. 86 (1964), 751-798.

9. A. Malcev, On a class of homogeneous spaces, Izv. Akad. Nauk. Armyan. SSSR Ser. Mat. 13 (1949), 201-212.

10. D. Mcduff, Examples of simply connected symplectic non-Kählerian manifolds, J. Differential Geom. 20 (1984), 267-277.

11. K. Nomizu, On the cohomology of compact homogeneous space of nilpotent Lie group, Ann. of Math. (2) 59 (1954), 531-538.

12. W. P. Thurston, Some simple examples of symplectic manifolds, Proc. Amer. Math. Soc. 55 (1976), 467-468.

Department of Mathematics, Faculty of Education, University of Nigata, NigataSHI 950-21, JAPAN 